\title{
BIODIVERSIDAD ALGAL DE LAGUNAS COSTERAS EN LA REGION CENTRAL DEL PERU ( Departamento de Lima )
}

\begin{abstract}
RESUMEN
Los esosisiemas acú́rions costeros de la zona destetica central del Pend evaluabos entre los $11^{\circ} 10^{\circ}$ y $12^{\circ} 32^{\prime} S$ incluyen las lagunas Puerto Viejo, Veatanilla none. Paraico, Huscho y Veatanillas sur. 5 e realizaron muestreos para la cvaluación de la flora algal y andissis de algumos parimetros fisice quîmikos (salinidad, $\mathrm{pH}$, temperatura) entre 1988. 1994. Rangos amplios de salinidad y pH esconseron entre 0-350 obo (salurseica de NaCl) y 6.5 . 10 respectivameme La fora algal estuvo constieuids principolmente por comunidades plancténicas, bentónicas, epibsicas, epifftas y endoliticas. Las clanobacterias osestituyen el grupo dominante con especies estene y surihakinas y con algunos flagelados fueron distribaidos de acuerdo al grado de tolerancia a ta salinidad. Adaptaciones morfológicas y ecológicas de algas en poblaciunes nararaies complementadas con los caltivos unialoales permite comprendet la biodiversidat aleal se ésios ecosistemas.
\end{abstract}

\section{SUMMARY}

Coastal aquatic ecosystems from central Peruvian desert area hetwoen $11^{\prime} 10$ and $12^{*}, 32^{\prime} S$ include Puerto Viejo, Higica. Ventanilla none, Paraiso, Villa, Huacto, Ventanilla Sur superficial lagoons. Sampling of these andland wikers for algal flock evaluation and physico-diemical analyses (salinity, pH, teroperatare) were carried out berween 1988. 1994. Algal Hora was typified for plankidonic, benthonic, epiroic, opiphytic and endelithic assemblages. Wide ranges of salinily and $\mathrm{pH}$ were berwen 0.350 a/oo (saturution NaCl) and 6.5-10 respectively. Flourishing popalations of cyanobscteris with eurytaline and stenohaline species as well as same flagellases vere disaribuied accoeding to the salinity iolerance. Algal morphological and ecologicel adaptations in narunil populations compiemented with thase in unialgal cultures let ux understanding the algal
biodivenity of these ecosystems.

\section{INTRODUCCION}

Los ecosistemas acuáticos costeros son de amplia distribución en las regiones áridas del mundo y de importancia desde el punto de vista económico, cientifico y ecológico (trofo-dinámico) porque el stress fisiológico en la biota es acentuado y acarrea adaptaciones extremas para la supervivencia. Entre los desienos costeros del oeste de Sudamérica, el desierto peruano forma una faja costera continua al oeste de las vertientes de la Cordillera de los Andes que tipifica al Perú localizado en la región tropical sur. En general, la topografia costera es baja y plana con una notable homogeneidad térmica que resulta en parte por la influeacia de la Corriente Peruana de Humboldt. En la Costa central es posible reconocer comunidades vegetales como las lomas y los gramadales como islas florísticas en el desierto hiperárido (RUNDEL et al., 1991).

El sistema hidrológico de la Costa Peruana comprende una variedad de cuerpos de agua tropicales, subtropicales y talasobalinos, con gradientes de salinidad (aguas dulces, salobres y salinas) asf como la presencia de suelos pantanosos (humedales) en áreas relativamente restringidas a poca distancia de la línea costera. Los ríos costeros originan reducidos valles agricolas que interrumpen la topografia árida de la región.

Las microalgas constituyen una fuente genética de gran potencial para mantener el desarrollo de la biosfera y en los ecosistemas acuáticos costeros son los principales contribuidores en los ciclos biogeoquímicos indispensables para la circulación de la materia orgánica. Investigación biosalina con estudios sobre las propiedades de las especies algales que habitan tales ambientes considerando el tipo de compuesto y mecanismos fisiológicos como base fundamental de la halotolerancia de las algas que explica su florecimiento se han realizado a nivel mundial (BROCK, 1975, HELLEBUST, 1976, OREN \& SHILO, 1982), En general, la influencia de la salinidad y la temperatura en la distribución, crecimiento y abundancia del fitoplancton en los extremos de los rangos tolerables es de vital importancia para una especie determinada (TOMAS, 1978). 
El estudio florístico de las microal gas de éstos tipos de ambientes y la estimación de su potencial en nuestro pais es muy limitado, solo tenemos referencia acerca de la presencia de algunas especies algales $y$ las condiciones físico-quimicas de las lagunas de Villa, Chilea y Huacho (MALDONADO, 1943, CHACON, 1980, MONTOYAet al., 1988). Debido a la relevancia de la biodiversidad biológica microalgal en el funcionamiento y mantención de éstos ecosistemas acuáticos se ha evaluado parcialmente las comunidades algales de la región central del Perú (Departamento de Lima) determinando su composición floristica aś como ci rango de tolerancia a las gradientes de salinidad $(\mathrm{NaCl})$ y $\mathrm{pH}$ que permitiría utilizar las especies algales como bioindicadores de éstos ecosistemas.

\section{MATERIALES Y METODOS}

Las lagunas costeras del departamento de Lima que tipifican determinadas localidades de la zona desértica costera fueron muestreadas en forma irregular así como los pequéోos cuerpos de agua (pozas, charcas) alcdaños a ellas entre 1988 y 1994. Las lagunas estudiadas se localizan entre los $11^{\circ} 1 \gamma^{\circ}$ y $12^{\circ} 32^{\circ}$ de latitud sur y fueron de norte a sur: Paraíso, Huacho, Ventanilla (norte y sur), Villa, Hipico y Puerto Viejo. Las lagunas talasohalinas como las de Huacho y Ventanilla sur son de origen marino mientras que las lagunas como Puerto Viejo, Paraiso y Ventanilla norte $s 0 n$ abastecidas con aguas dulces provenientes de sistemas fluviales e irrigaciones agricolas.

El reconocimiento de los diversos habitats algales y colecciones de las comunidades bentónicas fueron realizadas por remoción del substrato mientras que las planctónicas fueron transportadas en su medio natural. Las comunidades endoliticas fueron obtenidas de los alrededores de los cuerpos de agua constituídos por suelos calcárco-arenosos, costrosos, de perfil ondulado con cavidades y fisuras. Las endoliticas sumergidas fueron extraidas del interior del substrato sumergido en los cuerpos de agua. Las muestras frescas fueron llevadas. directamente al laboratorio para su observación microscópica. Parte de cada una de las muestras colectadas fueron fijadas y preservadas en formalina al $5 \%$ o en una solución de lugol. Registros de temperatura (termómetro de $-10^{\circ} \mathrm{C}-110^{\circ} \mathrm{C}$ ), salinidad (salinómetro A.O. T/C) y $\mathrm{pH}$ (papel y varillas indicadoras: 6.5-10; 4 7,alkalit 7.5-14) fueron realizados simultáneamente con las colecciones algales. La identificación de las especies fueron realizadas mediante consulta bibliogrifica especializada (GEITL.ER, 1932; ROUND, 1971; PRESCOTT. 1978: SILVA 1982: HINDAK, 1989).

Los cultivos unialgales fueron obtenidos en los medios $/ 2$ (GUILLARD, 1935) con $\mathrm{pH} 7$ y 35 ofoo de salinidad ( $\mathrm{NaCl}$ ) y N8 (usado en el Instituto del Carbón
Biologico de Dortmund, Alemania) eon pH 6.1 y 0 o/oo de salinidad $(\mathrm{NaCl})$. lluminación mediante támparas fluorescentes $40 \mathrm{~W}$ fue proporcionada así como iluminación natural a través de una ventana. Inoculaciones standard y técnicas de subcultivo fueron empleadas para mantener cultivos unialgales en medio líquido y agarizado a aproximadamente $21^{\circ} \mathrm{C}$.

\section{RESULTADOS}

\section{AREA DE ESTUDIO}

El desierto costero que constituye aproximadamente el $10 \%$ del territorio peruano es de geomorfología plana evidenciado por numerosas pampas de arena con dunas movedizas. En la parte central (departamentos de Ancash y Lima) la Cordillera de los Andes se acerca al mar formando una sucesión de acantilados rocosos por las estribaciones andinas a excepción de aquellos tramos por donde discurren los ríos costeros. Las condiciones atmosféricas proporcionan un clima desértico húmedo, moderado y uniforme. La parte central se caracteriza por usa estable nubosidad en invierno mientras que en los meses de verano se presenta un clima claro y luminoso con ocasionales nubes. Las temperaturas medias oscilan entre 19 y $23^{\circ} \mathrm{C}$ con precipitaciones egaruass en el invierno en el centro y sur.

Las lagunas costeras preferentemente en depresiones del terreno constituyen cuerpos de agua (dulces, salobres y salinas) superficiales (2,5 m de profundidad) alimentadas por aguas subterráneas que ascienden por capilaridad hasta la superficie donde al evaporarse pueden dejar sales como cloruro de sodio y sulfato de calcio (yeso) mezclado con una proporción elevada de arena eótica. Manantiales de aguas dulces y ligeramente salobres entre los espigones naturales de arena se atribuyes a percolaciones de aguas de rios durante las crecientes relacionados con las lluvias de verano en la región andina (Sierra), El suministro de aguas subterráneas en parte de la región desértica costera parece representar agua fósil introducida durante los periodos pluviales del Pleistoceno. Adiciones contemporáneas de significantes abastecimientos de agua son limitadas a inundaciones stubitas en los canales ribereños y están relacionados con fuertes lluvias asociadas con el fendimeno del Nifío (GOUDIE \& WILKINSON, 1977: RUNDEL et al., 1991).

\section{Lagunas de Puerto Viejo}

El sistema hidrológico compreade un grupo de lagunas superficiales eutróficas que se localizan a $70 \mathrm{~km}$ al sur de la ciudad de Lima entre las coordenadas $12^{\circ} 32^{\prime}$ de latitud sur y $76^{\circ} 45^{\prime}$ de longitud ceste, con una superficic aproximada de 1,000 ha y rodeadas en una gran extensión por las estribaciones occidentales de los Andes. El recurso hídrico proviene de la percolación de aguas subterráneas 
dulces probablemente de las cuencas de los ríos Chilca y Mala La salinidad de las aguas oscila entre 0-12 doo (NaCl) y el pH de 7,4-10, lo que revela la influencia de las sales cíclicas marinas por la cercanía del Océano Pacífico (aproximadamente $200 \mathrm{~m}$ ).

\section{Lagunas de Ventanilla}

Las lagunas de Ventanilla localizadas al norte de la ciudad de Lima a la altura del $\mathrm{km} 12$ de la Carretera a Ventanilla , eatre la coordenadas $11^{\circ} 52^{\prime}$ de latitud sur y $77^{\circ} 08^{\prime}$ de longitud oeste están rodeadas de cerros y colinas de poca altura correspondiente a las vertientes occidentales centrales de los Andes, El sistema hidrológico comprende lagunas superficiales del ladis norte (Veatanilla norte) en relación al desvió que conduce a la playa Ventanilla. Estas lagunas de fondo fangoso con aporte fluvial de aguas freáticas provenientes de la cuenca del rio Chillón (ROJAS, 1975) se caracterizan por la presencia de aguas con rangos de salinidad de $0-7$ oloo y pH de 3 - 10. Las lagunas del lado sur (Ventanilla Sur) son talasohalinas con filtraciones de agua marina por debajo de la barra o cordón litoral de arena y grava que impide la visualización directa de la playa. El rango de salinidad oscila de 6 - 150 oloo hista la saturación (salmuera) y los valores de $\mathrm{pH}$ de $7-8,7$. El frente de encuentro de las aguas subterraneas de mar y dulce en el litoral es variable a to largo del año lo que explicaría las variantes de salinidad existentes en áreas restringidas de Ventanilla.

\section{Laguna de Villa}

La laguna de Villa se encuentra localizada en el departamento de Lima, entre las coordenadas $12^{\circ} 12^{\prime}$ de latitud sur y $76^{\circ} 59^{\prime}$ de longitud oeste. Este ecosistema de aguas superficiales en su mayoría de tipo circulante es alimentado con aguas freáticas duloes que provienen de las filtraciones del río Surco que constituye un ramal del río Rímac y que desemboca en el Océano Pacifico. El rango de $\mathrm{pH}$ oscila de 6,8-9,5 y el de salinidad entre 0-14 ooo En casos excepcionales se detecto pozas de 142 ovoo y 194 ofoo de salinidad al borde de la laguna que podría explicarse por la presencia cercana de algún canal de filtración de agua marina suplementada con la salinidad del terreno.

\section{Lagunas de Huacho}

Las Lagunas salinas de Huacho están localizadas a 110 $\mathrm{km}$ al norte de la ciudad de Lima entre los $11^{\circ} 10^{\prime}$ de latitud sur y $77^{\circ} 30$ de longitud oeste, aledañas a la salinera más grande del paŕs, con un promedio anual de precipitación de 0-12,3 mm. Los cuerpos de agua talasohalinas por percolación de agua marina a través de la barrera costera son superficiales y permanentes en su mayoria. Algunos de ellos ilegan ser reducidos en volumen periódicamente dejando pozas y charcas temporales que se secan a fines de verano y otol̂o (PETERSEN, 1977). Debido a las variaciones climáticas se presentan oscilaciones en el nivel de agua acompanadas por cambios en gradientes salinidad siendo el rango de $80-350$ o/oo ( $\mathrm{NaCl}$ ) y el pH de 6.5-8. La temperatura del agua osciló entre 22 y $36^{\circ} \mathrm{C}$.

\section{Laguna EI Paraiso}

La laguna costera El Paraiso (Playa Chica) está localizada entre las coordenadas de $11^{\circ} 12^{\prime} \mathrm{S}, 77^{\circ} 36^{\prime} \mathrm{W}$ a $10 \mathrm{~km}$. al sur de la ciudad de Huacho. El nivel de agua está influenciado por las mareas de la playa aledaña y por el agua proveniente de las filtraciones de los terrenos de cultivo próximos (CASTRO et al., 1990). Sus aguas evidenciaron un rango de $\mathrm{pH}$ de 9.9 .5 y gradiente de salinidad de 0-20 o/oo.

\section{Pozas del Hípico}

Las pozas del Hípico localizada aproximadamente a $500 \mathrm{~m}$ al sur de la laguna de Villa comprende cuerpos de agua pequeños y charcas con un rango de salinidad de 4122 oloo y valores de $\mathrm{pH}$ de 8,5-10. Al parecer el sistema hidrológico proviene de la filtración de aguas freáticas similar al de la laguna de Villa y filtración de agua marina por las amplias variaciones en salinidad en pequeñas áreas.

\section{DIVERSIDAD ALGAL}

La diversidad de la flora algal de las lagunas costeras del departamento de Lima correspondiente a la Costa central esta constituida principalmente por comunidades planctónicas, bentónicas epizoicas, epifitas, saxícolas y endoliticas. Se reportan las comunidades más representativas con la especie dominante, su rango de salinidad, $\mathrm{pH}$ y especies asociadas. Los ambieates acuáticos evaluados con un rango de $\mathrm{pH}$ de 6,5-10 oscilan entre los de agua dulce y los hipersalinos basta la saturación de $\mathrm{NaCl}(350$ o/ $/$ ). Los grupos y especies algales de las lagunas costeras evaluadas del departamento de Lima fueron:

\section{PHYLUM CYANOPHYTA}

\section{CYANOPHYCEAE}

Chroococcales Chroococcaceac Gomphosphaeria aponina Kuetring Merismopedia glauca (Ehr.)Naegeli Microcystis aeriginosa Kuetzing Aphanothece halofitica Fremy Aphanothece stagnina A.Braun Chroococcus dispersus Lemmermann Chroococcus Limnericus Lemmermann Chroococcus turgidus (Kwetz) Naegeli Chamaesiphonales

Pleurocapsaceac Pleuracapsa entophysaloides Setchell \& Gardner 


\author{
Oscillatoriales \\ Oscillatoriaceae \\ Lyngbya martensiana Meneghini \\ Spiralina sabsaisa Cersted \\ Oscillatoria amphibia C.A.Agardh \\ Oscillatoria princeps Vaucher \\ Oscillatoria tenuis C.A.Agardh \\ Oscillatoria formosa Bory \\ Microcoleus chrhonoplastes (Mert) Zanardini \\ Phormidium sp. \\ Nostocales \\ Nostocaceae \\ Anabaenopsis elenkinii Miller \\ Anabotena variabilis Kuctzing \\ Nodularia harveyana (Thw.)Thuret \\ Nodularia spumigena Mertens \\ Rivulariaceae \\ Gloeotrichia natans (Hedwig) Rabenh. \\ Calothrix sp.
}

\section{PHYLUM CHLOROPHYTA}

\author{
CHLOROPHYCEAE \\ Volvocales \\ Dunaliellaceac \\ Dunaliella salina Teodoresco \\ Dunaliella viridis Teodoresco \\ Chlorococcales \\ Oocystaceac \\ Oacysis eremosphaeria G.M.Smith \\ Chiorella sp. \\ Scenedesmaceac \\ Scenedesmus quadricauda (Turpin) de Breb. \\ Hydrodictyaceac \\ Pediastrum boryanum (Turpin) Menenghini \\ Dictyosphacriaccae \\ Botryococcus brawti Kuctzing \\ Zygnematales \\ Zygnemataceae \\ Ulvales \\ Spirogyra sp. \\ Ulvaceae \\ Enteromorpha prolifera (Mueller) Agardh \\ Enteromorpha sp. \\ CHAROPHYCEAE \\ Charales \\ Characeae \\ Chara sp.
}

\section{PHYLUM PRASINOPHYTA}

\section{PRASINOPHYCEAE \\ Prasinocladales \\ Tetraselmiaceae \\ Terraselmis $\mathrm{sp}$.}

\author{
PHYLUM EUGLENOPHYTA \\ EUGLENOPHYCEAE \\ Euglenales \\ Euglenaceac \\ Euglena gracilis Klebs \\ Lepocinclis fusiformis (Carter) Lemm
}

\section{PHYLUM CRYPTOPHYTA}

\section{CRYPTOPHYCEAE \\ Cryptomonadaceae \\ Cryptomonas sp.}

\author{
PHYLUM PYRRHOPHYTA \\ DESMOKONTAE \\ Desmomonadales \\ Prorocentraceae \\ Exuviaella cassubica Woloszynska
}

\section{PHYLUM CHRYSOPHYTA}

\author{
BACILLARIOPHYCEAE \\ Pennales \\ Nitzchinceae \\ Nitzschia closterium \\ Surirellaceac \\ Campylodiscus sp.
}

\section{PHYLUM RHODOPHYTA}

\author{
Bangiales \\ Erythrotrichaceae \\ Compsopogon coenuleus (Bailbis) Montagne
}

\section{COMUNIDADES ALGALES}

\section{Comunidades bentónicas}

- Formando mantos gelatinosos o natas sumergidas azul verdes oscuros, verde parduzcos o pardo amarillentos, tapizando el fondo fangoso de las orillas de lagunas y pozas que en muchos casos presentan bacterias azufradas. Estas comunidades constituidss principalmente por la cianofita filamentosa Spirulina subsalsa con un rango de salinidad de $0-130$ oloo y $\mathrm{pH} 6,8-10$, pueden desprenderse como natas o películas densas flotantes. En ambientes acuáticos de bajas salinidades $(0-7$ o/ oo; $\mathrm{pH} 9$ 10) como los de Ventanilla norte coexiste con Lyngbya martensiana, Nadularia spumigena, N.harveyana, Scenedesmus quadricauda, y Oscillatoria tenuis. Entre las salinidades de 2-5 ofoo y pH: 9,5-10 en pozas del Hípico $S$ s subsalsa se presenta planctónica asociada con Euglena gracilis, Chroococcus dispersus y Chlorella sp. A mayores salinidades en el Hifico (10-22 o/oo; $\mathrm{pH}: 9.5$. 10) coexiste con Calothrix sp. formando biodermas verde 
oscuro o parduzcos, de textura gelatinosa-coriacea con proyecciones de contorno ondulado y babitat saxícola (epilftica). En ambientes hipersalinos (85-130 o/oo; pH: 6,5-7) como los de Huacho forma poblaciones densas asociadas con O.tensis, O.amphibia, Aphanothece halophytica, Tetraselmis sp. y diatomeas. La cepa de Ventanilla norte fue posible aislarla en medio N8.

- Masas grumoso-gelatinosas formando mantos sumergidos que se desprenden como natas flotantes espumosas (burbujas de oxigeno atrapadas) en pozas formadas en bordes de eflueate de laguna de Ventanilla norte de aguas dulces $(0 \mathrm{voo} ; \mathrm{pH} ; 7,7-8,1)$ que van a desembocar en la playa de Ventanilla ubicada a aproximadamente $100 \mathrm{~m}$ de distancia. A esta comunidad de agregados coloniales de Gomphasphaeria aponina como especie dominante puede considerarse que alcanza condiciones óptimas que permiten su proliferación por su alta densidad colonial en verano $\left(27^{\circ} \mathrm{C}\right)$. Coexiste con S.subsalsa, O.tenuis, $C$. turgidus, S.quadricanda y Spirogra sp. En menor proporción $G$. aponina se encuentra en aguas con salinidades de $5-10$ o/oo y pH: 7,4, 9.5 - 10 (Villa, Puerto Viejo) asociada con C. murgidus, C.dispersus, O.senuis, O.princeps, Microcystis aeruginasa, Exaviaella cassubica, Campylodiscus sp. y Chara sp.

- Formando natas o mantos sumergidos verde oscuro, pardo verdoso o verde claro en lagunas y pozas entre totorales que pueden desprenderse del sustrato de fondo lodoso (epipelica) originandis capas gelatinosas flotantes. Esta comunidad estuvo dominada por cianofitas. filamentosas con predominancia de Lyngbyamartensiand en un rango de salinidad de 0-17 $0 / 00$ y pH 7,4-10 (Puerto Viejo, Ventanilla norte, Hípico). Asociada principalmente con O.tensis, N.harveyana, N.spumigena, Ssubsalsa, $C$. dispersus y Phormidium sp. Cultivos de $L$ martensiana de 20 dias en medio N8 tuvieron una proliferación notoria comparado con el escaso crecimiento en medio $f 2$.

Formando mantos gelatinosos a coriáceos de superficie irregular originandos por colonias adyacentes que se fusionan como un substrato verrucoso continuo pardo amarillento, pardo oscuro o verde azulado oscuro firmemente adherido a substrato salino calcáreo. Esta comunidad bentonica tipificada por la cianofita cocoide colonial Pleurocapsa entophysaloides es dominante en lagunas salinas de Huacho (80-190 o/oo; $\mathrm{pH}: 6,5-8)$ y Ventanilla sur ( $80-145$ oloo; pH: 6,5-7,5). Sin embargo pucde tolerar mayores salinidades como se reconoció en lagunas hipersalinas (salmueras: $350 \mathrm{o} / \mathrm{o}$ ) asociada con $O$. tenuis y $O$. amphibia.

- Formando biodermas como mantos algales verdosos tapizando el bentos con la cianofita dominante Microcoleus chthonoplastes de talo filamentoso agregados por mucflago colonial que consolida el bentos calcśreo arenoso evitando su erosión (Paraiso : $\mathrm{pH}$ 9.9,5; $20 \mathrm{o}(\infty)) . M$. chthonoplastes también se presentan asociada con otras cianofitas filamentosas como Lyngbya sp. proporcionado el aspecto de un mosaico algal.

- Formando natas flotantes, verde claras, verde oscuras, espumosas en pozas de borde de laguna y en áreas pantanosas, con rango de salinidad de $0-7$ oloo y pH de 7,0-10. Comunidad tipificada por la clorofitaScenedesmus quadricauda que se presenta en Ventanilla norte $(0-7 \mathrm{o}$ oo; pH: 7-10) y Puerto Viejo (2 oloo; pH: 7,9-8,1) asociada principaimente con $C$. dispersus, $N$, spumigena, $N$. harveyana, S. subsalsa, $O$. tenuis, $L$ martensiana, Anabaenopsis elenkinii y Anabaena variabilis. En aguas dulces de Ventanilla norte $(0$ ofoo; $\mathrm{pH}: 7-7,5)$ se presenta preferentemente ascoiada $\operatorname{con} G$. aponina, Pediastrum bonyanam y Spinalina subsalsa. La cepa de $S$, queadricauda de Ventanilla norte fue aislada en medio agarizado N8 formando colonias redondeadas verde oscuras.

- Floraciones de la prasinofita Tetraselmis sp. en lagunas, pozas y charcas de orilla de lagunas y en manantiales, con un rango de salinidad de $6-155 \propto / \infty 0$ y $\mathrm{pH}$ ; 6,5-10. En la mayoría de éstos cuerpos se reconoció contaminación orgánica por la fertilización natural ocasionada por aves y el ganado (vacuno, equino, caprino). Forman películas superficiales densas, verde amarillentus, en algunos casos espumosas (Puerto Viejo, Huacho) y masas suspendidas verde amarillentas o pardo amarillentas (Hipico, Villa), verdosas (Huacho, Ventanilla sur). Las cepas de Tetraselmis sp. de baja salinidades como en Puerto Viejo (6-8 o/oo; pH: 8-10) coexiste principalmente con el dinoflagelado Exuviaella cassubica, ef euglenoide Euglena gracilis y la diatomea Campylodiscus sp. En mayores salinidades como en el Hípico (38-54 o/oo) se presenta asociada con Chlorella sp. y diatcmeas. En los cuerpos de aguas hipersalinas de Ventanilla sur (65-70 ovoos pH: 8-8.5), Hípico (100-122 ov $\infty$; pH: 8,6-10). Villa (130-142 o/oo; $\mathrm{pH}: 9,5)$ y Huacho (88-155 oloo; $\mathrm{pH}: 6,5-8$ ) coexiste con Dunaliella viridis, $O$. tenais, Aphanothece stagnina y $A$. halophyrica. En Huacho se ha observado condiciones optimas para Tetraselmis sp, por la altat densidad poblacional en los meses de verano entre 130 y 145 o/oo de salinidad $(\mathrm{NaCl})$.

- Formando natas azul verdes densas, flotantes y sumergidas en pozas con aguas de consistencia mucilaginosa y capas superficiales cristalizadas. Exhiben un rango de salinidad de 130-142 $0 / 00$ y pH: 9,5 (Villa). Esta comunidad tipificada por la cianofita colonial dominante Aphanothece stognina podtía considerarse en condiciones óptimas por su alta densidad poblacional. Esta asociada con $O$. formosa. Tetraselmis sp. y diatomeas. En las lagunas de Puerto Viejo y Ventanilla norte con salinidades de 6-7 ofoo y pH: 9-10. A. sfagnina se halla en minima proponción juato con otras cianofitas. Las cepas de A. stagnina de Puerto Viejo, Villa y Ventanilla norte con células vegetativas elipsoidales u ovoides, embebidas en el mucilago colonial denso, difluente e irregular $50 n$ similares. 
a las de $A$. halophytica de Huacho (91- 155 ofoo; $\mathrm{pH}: 6,5-8)$ y Ventanilla sur (75-145 doo; $\mathrm{pH}: 6,5-7,5)$ con excepción de la delimitación del mucflago que tipifica a las colonias esféricas de A. halopityfica. En diversos ambientes acuáticos en Huacho $A$. halophytica prolifera temporalmente (verano y otoho) coexistiendo con Tetraselmis sp., $S$ subsalsa, $O$. tenuix y diatomeas.

- Floraciones de estadíos unicelulares mótiles de Duonaliella viridis con formación de películas verde amarillentas colonizando ambientes de amplio rango de salinidad como en Ventanilla sur (65.150 o/o0; $\mathrm{pH}$; 7 8,7), Hipico (90-122 doo; pH: 10), Huacho (80-350 doo; $\mathrm{pH}: 6,5-8)$ y Villa $(194$ o/oo). El estado béntico palmeloide durante el ciclo de vida de $D$. viridis forma masas gelatinosas verdosas en Huacho y Veatanilla sur $(50 \mathrm{ovo})$. En Huacho se presenta asociada con el estado palmekide de $D$. salina en las lagunis y pozas hipersalinas que alcanzan la saturación ( 350 o/oo). $D$. salina se presenta libre como masas en suspensión rojo ladrillo siendo el estado palmeloide mucilaginoso de coloración rojiza en Huacho (165-350 o/oo; pH: 6,5-8),

- Formando natas gelatinosas azul verde flotantes y sumergidas en lagunas y pozas con vegetación macrofitica dominada por Chara sp. y con un rango de salinidad de 56 oroo y pH: 7.5-9.5 (Puerto Viejo, Villa). Comunidad constituida principalmente por la cianofita cocoide Chroococcus dispersus asociada con Microcystis aeruginosa, C. turgidus, C. limneticus, Merismopedia glauca y Phomidium sp. La ckscofita Oocystis eremosphaeria y la cianofita Gloeotrichia natans también estuvieron presentes en Puerto Viejo y la segunda especic ademśs en Villa. $C$ dispersus y $C$. linneticus de Ventanilla norte (0-7 o/co; $\mathrm{pH}: 9-10)$ aunque no llegan a ser dominantes constituyen parte de la comunidad bentónica junto con las cianofitas $S$. subsalsa, $N$. harveyana y $N$. spamigena. La cepa de C. dispersus de Puerto Viejo fue aislada en medio $\$ / 2$ con el desarrollo de colonias esféricas verde oscuras a verde olivo y también en medio N8 con colonias mas bien laminares de una coloración verde azulada intensa to que evidencia sa naturaleza eurihalina luego de un periodo de adaptación (1 mes).

- Floraciones algales del fiagelado Euglena gracilis formando masas suspendidas de tonalidades verde ascuras o verde amarillentas en lagunas y pozas con un rango de salinidad de 2-22 ofoo y pH de 8-10. E. gracilis en altas densidades (Puerto Viejo. Hípico) origina natas o peliculas espumosas en ambientes con rango de salinidad de $2-8$ o/oo. Frecuente en lugures con contaminación orgánica por la fertilización natural de aves y ganado (Hípico, Puerto Viejo). En mecoor proporción estuvo presente en Ventanilla norte $(6-7$ do\%; $\mathrm{pH}: 10)$. Se presenta asociada con Chiorella sp.. Exuviaella cassubica, C. dispersus, S.subsalsa y Tetraselmis sp.
- Floraciones del dinoflagelado Exaviaella cassubica que forma poblaciones densas proporcionando tonalidades parduzcas al cuerpo de agua y llegando a formar peliculas superficiales en pozas y lagunas con un rango de salinidad de 2-15 o/oo y pH: 7,9-10 (Ventanilla norte, Hipico, Puerto Viejo), Asociada con C. dispersus, $E$. gracilis, Tetraselmis sp. y Cryptomonas sp.

- Formando masas verde-amanillentas, verde oscuras. flotantes y sumergidas de la clorofita filamentosa Enteromorpha $\mathrm{sp}$. con talo cilíndrico rugoso y contorneado, en borde de lagunas y charoos de Huacho (88-110 o/oo; pH: 6.5-8). Hípico (12-22 ovo; pH: 8,8-10) y Villa $(12-14$ o/oo; $\mathrm{pH}: 7,8$ ). El talo originalmente saxicola también sirve como substrato para el desarrollo de epifitos cono Pleuracapsa entophysaloides en Huacho.

- Floraciones temporales de la criptofita Cryptomonas sp. proporciona una coloración pardo amarillenta al cuerpo de agua (Paraiso: pH: 9-9,5; 0-10 o/oo) asociada con especies subdomisantes como Nitzschia closteriam y Gomphosphaeria apanina a comienzos de primavera. Posteriormente se evidenció la sucesión algal con proliferación de la cianofita Plearocapsa sp.

- Foraciones de la clorofita Botrococcus brainiz en lagunas de Puerto Viejo $(\mathrm{pH} \quad 6,5-8,3 ; 0-10$ o/oo) constituyen masas coloniales agregadas por conexiones mucilaginosas asociadas principalmente con Exwriaella cassubioa, Lepocinclis furifonmis, Lyngbya mantensiana y Anabiena sp.

\section{Comunidades epizoicas}

- Densas masas de algas verdes filamentosas como Enteromorpha prolifera colonizan las valvas de moluscos como Sinum cymba (Naticidae), ostras y Balanus sp. en la lagunas del Paraiso. E prolifera también puede presentarse formando comunidades epifitas (hidrofitas superiores) y saxicolas, En Ventanilla norte $(\mathrm{pH}: 7,1-7,4)$ coloniza las valvas de Melanoides faberculata donde se presenta asociada con Compsopogon coerileus y Stigeoclonium sp. Esta útima especie que también forma capas verdosas amarillentas sobre las valvas de los moluscos puede presentarse en forma saxicola.

\section{Comunidades endolíticas}

- Comunidades cripto y easmoendolitica con colonias de Pleurocapsa entophysaloides habitando cavidades estructurales y fisuras pre-existentes en suelos aledaños a lagunas (Huacho y Ventanilla sur) llegando a ser incluidas en la matrix cristalina. traslucente y porosa. Crecimiento algal como estratos azul verdes de $0,2-2,0 \mathrm{~cm}$ por debajo de la superficic 
costrosa. También puede presentarse inregularmente distribuida y asociada con $O$. tenuis y $O$. amphibia.

- Formando natas o capas gelatinosa-membranosas irregulares, verde parduzca o azul verde oscuras. tapizando el interior del sustrato de fondo constituído por sales cristalizadas en lagunas hipersalinas de Huacho de (165-350 ofoo; pH: 6,5-7). Esta comunidad endolítica sumergida esta dominada por la cianofita filamentosa Oscillatoria tenuis y en menor proporción por $O$. amphibia.

\section{DISCUSION}

Debido a las variaciones climáticas de la Costa Peruanacomo lasescasas precipitacjones que no permiten el drenaje de las sales del suelo y las que se forman por los agentes atmosféricos (transporte de sales cíclicas vía eólica) las lagunas costeras están sujetas a un incremento en salinidad aunada a la desecación estacional de sus litorales con pozas efimeras aledafias. La presencia de determinadas especies algales que requiere en algunos casos la inoculación de poblaciones autóctonas que fueron capaces de resistir períodos de desecación (estados de resistencia en su ciclo de vida) es consistente con su distribución aparentemente éptima cuando originan floraciones algales demostrando que la salinidad y pH tienen diferentes efectos en su sobrevivencia y crecimiento. Las comunidades algales constituidas en sa mayoria por especies eurihalinas de las lagunas Puerto Viejo, Ventanilla norte, Villa y pozas de. Hípico son más ricas en especies que las lagunas talasohalinas (Huacho, Ventanilla sur) corroborando que la distribución y la diversidad biótica de las especies esta inversamente correlacionado con la salinidad (VARESCHI, 1982, COMIN et al., 1983). Los rangos de tolerancia algal a la salinidad y $\mathrm{pH}$ ballados en las lagunas costeras esti en relación con la dinámica de las poblaciones naturales y la biogeografia de las especies que permite tipificarlas como bioindicadores para la región costera peruana.

La tolerancia a un amplio rango de salinidad fue demostrado en cultivos de algas bénticas litorales en lagos alcalinos salinos (Lagos Mono y Abert, USA) aunque las velocidades de crecimiento y almacenamiento (contenido orgánico) fueron limitados por el incremento de salinidad dentro del rango óptimo (HERBST \& BRADLEY, 1989). La dominancia de las comunidades algales bénicas por cianobacterias en nuestros lagos como matas cohesivas creciendo sobre una considerable heterogeneidad espacial originada por el sustrato de fondo como superficies rocosas (algas epiliticas) o sedimentos fangosos (algas epipelicas) puede ser atribuida al rango de $\mathrm{pH}$ alcalino relacionada con iones de calcio y bicarbonato de aguas duras en suelos calcáreos como los de la costa Peruana. Además esta comunidad béntica contribuye en gran proporción a la biomasa algal planctónica por desprendimiento de los biodermas en zonas litorales. Entre las cianofitas bentónicas Spirulina subsalsa con un amplio rango de tolerancia a la salinidad $(0-130 \mathrm{\alpha} / \mathrm{oo})$ evidenció su naturaleza eurihalina formando extensos biodermas en habitats salinos ( $85-100$ o/oo; $\mathrm{pH}: 6.5-7$ ) que revela su óptimo crecimiento y avala su naturaleza halofitica. Sin embargo en aguas dulces y salobres ( $\mathrm{pH}: 9$. 10) se presenta en menor proporción probablemente debido a la competencia interespecifica 6 al pH alcalino.

La distribución de Nodilaria spumigena que en nuestras lagunas (Ventanilla norte, Hípico) es más bien litoral bentónica con un rango de salinidad de 0-17 $/ \%$ puede ser comparada con ta de los lagos salinos del área Saskatchewan (Canada) donde arigina floraciones monoespecificas en el lago Manito con un rango de salinidad de 22 a $24 \mathrm{~g} \cdot \mathrm{H}^{-1}$ (HAMMER et al., 1975), Según SMARDA et al.,1988) N. spumigena forma poblaciones planctónicas que desarrollan floraciones densas y también colonizan localidades extremas (suelo, pozas salinas, manantiales minerales, etc.).

En lagos tropicales el crecimiento de cianobacterias puede set através del año mientras que en regiones templadas existe desarrollo de floraciones estacionales como el de Microcystis aeruginosa (junio-octubre). Por ejemplo, el lago Humboldt (Canada) con $3,5-7 \mathrm{~g} \cdot \mathrm{t}^{-1}$ de salinidad presenta floraciones mixtas anuales de $M$. aeruginosa principalmente con Apharizomenon flosaquae y Stephanodiscas niagarae. Asi mismo las poblaciones naturales de $M$. aeruginosa en las lagunas (Villa, Puerto Viejo, Ventanilla norte, Hípico) con rango de salinidad de 4-12 doo estarían relacionadas con un requerimiento bajo de sodio para ésta especie según MCLACHLAN \& GORHAM (I961). Igualmente ta asociación de $M$. aeruginosa y otras especies con ta vegetación macrofítica de Chara sp, en las lagunas de Villa y Puerto Viejo, podría estar relacionada con la baja salinidad como en las poblaciones de Chara sp. del lago Wakau con rango de salinidad de $3,5-4,3 \mathrm{~g} \cdot \mathrm{l}^{-1}$. Por consiguiente, las diferencias fisiologicas entre especies algales permiten su colonización en cuerpos de aguas dulces y salobres, mientras otras requieren mayores salinidades como P. entophysaloides, A. halophytica, D. salina y $D$, viridis.

La agregación celular en poblaciones algales naturales parece ser un paso preliminar en la formación del estado béntico palmeloide en especies como $D$. viridis y $D$. salina donde la copiosa producción y excreción de carbotidratos es necesario para producir la matrix extracelular caracteristica del estadio béntico. La liberación de células del estado béntico puede ser un modo de restablecer poblaciones mótiles en ambientes favorables, como se evidenció adicionando $f / 2$ a las células palmeloides. Por lo tanto, la plasticidad morfologica relacionada con el estado béntico puede actuar como una fase de sobrevivencia algal cuando se colonizan lagunas costeras hipersalinas tropicales. 
El mejor conocimiento de las poblaciones algales, estructura de sus comunidades con la composición especifica y sucesión permitirá evidenciar la diversidad algal asi como sus interacciones biológicas en nuestros ecosistemas acuátieos costeros.

\section{AGRADECIMIENTOS}

Nuestro reconocimieato al $\mathrm{Dr}$. Pedro Huamán por la ideatificación de los moluscos, Roberto Quesquen y Victor H. Vera por su colaboración en las colecciones realizadas.

\section{BIBLIOGRAFIA}

BROCK. T.D. 1975. Salinity and the eoxlogy of Dunaliella from Great Salt Lake. J. gen.Microbial.89:285-292.

CASTRO, G., E. ORTIZ \& L. BERTOCHL 1990 Importancia biológica y conservación de la laguna El Paraiso, Lima. Bol. Lima. 71: 47.55.

CHACON, G.R., 1980. Chlorella peruviana sp. nov, $y$ su ambiente altamente salino. Bol.Soc.Per.Bot. 8:83-96. Lima.

COMIN,F.A., M.,ALONSO,P.LOPEZ \& M.COMEULES. 1983. Limnology of Gallocanta Lake. Aragon, northeastern Spain. Hydrobiologia. 105:207-221.

GETILER, L 1932 . Cyanophyceac, In Rabenhorst's Kryptogamenflora von Deutschland, Osterreich und der Schweiz Akad, Verlagsges. Leipzig. 14:1-1196.

GOUDIE, A. \& J. WILKINSON, 1977. The warm desert envirument.Cambridge Univ. Press. Cambridge. 88 p.

GUILLARD, R.L. 1975 Culture of phytopiankion for feedingmarine invertebrates. In W. L.Smith \& M.H. Chanley eds Culture of marine invertebrate animals. Plenum Press. New York.29-60 p.

HAMMER, U.T., R.C.HAYNES, J.M. HESELTINE \& S.M.SWANSON. 1975. The saline lakes of Saskatchewan. Verh Internat. Verein. Limnol. 19-589-598.

HELLEBUST, J.A, 1976. Osmoregulation. Ann.Rev. Plant Physiol. 27: 485-505.

HERBST, D.B.\& T.J. BRADLEY. 1989. Salinity and nutrient limitations on growth of benthic algae from two alkaline salt lakes of Western Great Basin (USA). $J$. Phycol. 25:673-678.

HINDAK.F., 1988.Planktic species of two related genera Cylindrospermopsis and Anabaenopsis from Western Slovakia. Arch Hydrobiol Suppl 80: 283-302.
MALDONADO, A, 1943. Las lagunas de Boza, Chilca y Huacachina y los Gramadales de la Costa del Perí. Act. Trab. 2do, Cong. Per. Quim. Lima. 143 p.

MCLACHLAN, J., 1961. The efects of salinity on growth and chlorophyll content in representative classes of unicellular marine algae, Can, J. Microbiol. 7:399-406.

MCLACHLAN,J. \& P.R.GORHAM,1961. Growth of Microoystis aeruginosa Kutz, in a precipitate free medium buffered with Tris. Can.J. Micnobiol, 7:869-882.

MONTOYA, H. C.BARBERENA \& R.QUESQUEN. 1988: Algas de las salinas de Huacho, Perú, con énfasis en Aphanothece halophytica Fremy. En H.Salzwedel \& A.Landa eds.Recursos y dinámica del coosistema de afloramiento peruano, Bol. Inst. Mar. Perú-Callas, Vol. extracordinario. $67-75 \mathrm{p}$.

OREN, A. \& M. SHILO, 1982. Population dynamics of Dunaliella parva in the Dead Sea. LimnoL. Oceanogr. 27 : 201-215.

PETERSEN, G., 1977. Historia maritima del Penú. Geografía y geología general del litoral peruano, Vol.I. Inst. Estudios Histónico-Marítimos del Perú. Lima. 214 p.

PRESCOTT, G.1978. How to know the freshwater algae.W.C. Brown Co. Publishers. Iowa. 293 p.

ROJAS, A.C., 1975. Estudio de los servicios e industrias en el área urbana de Ventanilla. Tesis. Universidad Nacional Mayor de San Marcos, Lima.165 p.

ROUND, F.E.,197L The taxonomy of the Chlorophyta II Br. Phycol.J. 6: 235-264.

RUNDEL.P.W ${ }_{4}$ M.O. DILLON, B.PALMA, H.A. MOONEY,S.L.GULMON \& J.R. EHLERINGER, 1991. The phytogeography and ecology of the coastal Atacama and Peruvian deserts, Aliso. $13 ; 1-49$.

SLVA, P, 1982 Thallobionfa. In Synopsis and Classification of living ofganisms. S.P. Parker ed. Mc Graw-Hill Book Co.133-161p.

SMARDA, J. J. KOMAREK, J. CASLAVSKA \& M. HUBEL 1988. The Nodularia studies, L. Introduction, fine structure. Arch. Hydrobiol.Suppl, 80:109-129.

TOMAS, C., 1978. Olisthodiscus luteus ( Chrysophycese) L. Effects of salinity and temperature on growth, motily and survival. J. Phycol, 14:309-313.

VARESCHI, E.1982. The ceology of Lake Nakuru (Kenya).III Abiotic factors and primary production. Oecologia 55:81-101. 